\title{
Improved Method of Springback Compensation in Metal Forming Analysis
}

\section{P. Bałon, ${ }^{1}$ A. Świątoniowski, ${ }^{2}$ and J. Szostak ${ }^{3}$}

AGH University of Science and Technology, Department of Manufacturing Systems, Kraków, Poland

\footnotetext{
${ }^{1}$ balonpawel@gmail.com

2 swiatoni@imir.agh.edu.pl

3 szostak@agh.edu.pl
}

Elements of automobile production are frequently fabricated from ultrahigh-or high-strength steels, which ensure their considerable weight reduction and high durability, but manifest a side effect called springback. The springback value depends mostly on the material used, as well as the geometry of part being formed. Thus, in extreme cases, the deviation value from the reference part may exceed the limiting value. Prediction of springback by the trial-and-error method and subsequent deviation correction is difficult and labor-intensive. Numerical compensation methods make it possible to obtain accurate results in a short time, which advantage eliminates unnecessary milling during die production and facilitates its production on a just-in-time basis according to customers expectations and forecasts for vehicle demand. This paper presents an innovative compensation method for both forming and trimming die for the construction of vehicle parts manufactured by a transfer press. This method allows one to optimize the accuracy of compensation, consequently reducing springback in a more exact way than currently used methods, which account only for the influence of trimming on springback without generating compensated surfaces for the trimming die and the next forming operation. Moreover, there is a possibility to include the positioning effect during multioperation forming because of the huge impact of results on separate operations. When the positioning is not considered between operations there are problems with a proper shape even if the final part has the correct geometry. These problems generate some additional costs during the die production, which can be avoided by using the multioperation compensation.

Keywords: springback, metal forming, multicompensation, simulation analysis, ultrahighstrength steel (UHSS), high-strength steel (HSS).

Introduction. Higher standards of comfort and safety have caused a continuous rise in the average vehicle weight and fuel consumption. Therefore, new solutions are still sought, in order to ensure the optimal mass of supporting structure and positive crash test results [1-3]. The creation of brand new steel grades with strong mechanical properties was a breakthrough innovation in steel research [4]. UHSS and HSS materials are the result of thorough research efforts conducted through joint international projects over the last decade. They have enabled the fabrication of high endurance materials preserving a good plasticity. Although these two parameters do not typically coexist and an increase in one of them is at the expense of the other, some material groups have the beneficial values of both.

These competitive properties and economic considerations are the main reasons that steel is the dominant material in the automotive industry. According to the forecast, in the next few years the percentage of UHSS and HSS materials in vehicle bodies is expected to reach about $70 \%$. These trends reflect the efforts for harmonization with the EU regulations requiring a reduction of overall structrue mass and simultaneous improvements in safety and fuel consumption [2].

In automotive applications, the numerical analysis is used to explore and predict forming possibilities in practice, in order to avoid some drawpiece mistakes. The potential 
problems in the stamping process during the tool design process can be eliminated by virtual fabrication software rather than by the trial-and-error process. This advanced and complex software enables a fast and reliable verification if the assumed method is correct, which gives an advantage over traditional trial methods, in a quite difficult prediction of the number and types of forming stages, the exact level of springback effect, which should take into account all inhibiting factors such as wrinkling, cracking, and, especially, the springback [5-7].

Springback compensation by means of tool geometry change must be necessarily situated within increasingly narrow tolerance intervals. The quality of stamped parts becomes more and more critical, since it influences the assembly stage and final product quality. One of the factors affecting the quality of drawpiece is shape-dimensional accuracy resulting from the material springback. Research conducted in recent years has narrowed of the tolerance range in constituent components of vehicles, as well as in assemblies. Body panels are designed in this way, in order to obtain high geometry stiffness. Their execution reaches the limiting values of possibilities for mass production.

Numerical calculations of forming are reduced to describe a surface with some elements that are based on nodes, the number of which should be sufficient for ensuring a good accuracy of calculations. Nowadays, compensation calculations are carried out in an automatic - or at least semi-automatic - way with the use of FEM software (e.g., PamStamp, Autoform, Ls-Dyna, Outifo, Mashal). This makes it possible to compensate the entire operation chain, which enables manufactures to take the springback into account for all process stages. It also provides an advantage in solving some problems involved with finding an accurate surface for cutting operations and matching the sheet to the tool during each individual operation. Foregoing the multioperational compensation tool requires the account of unstressed surfaces at particular trimming stations, which is not accurate, so it surely contains some fault or has some positioning problem [8].

The authors present an innovative method for multioperation compensation, which eliminates the problems of shaping the cutting tool surface and positioning of a blank. The authors have much experience in forming of vehicle structural elements and steel production, however, the springback experienced with multioperation tools often results in some problems, especially for HSS and UHSS steels. Therefore, new methods have been elaborated, which are different from both the trial-and-error and standard methods of numerical compensation, and yield brings better results. Compensation methods discussed in this paper are based on numerical models, while the resulting physical example is compared with the FEM models. In the numerical modeling, accurate material data were used so that the numerical analysis was totally compatible with the true case.

1. Die Compensation. The main idea of the die correction consists in application of classic springback compensation used in technological settings, i.e., overbending during forming, which enhances the springback elimination. For simple forming, the problem is reduced to the additional sheet overbending. However, if the drawpiece geometry is more complicated, it becomes quite problematic to predict the material behavior. Nonlinear behavior during the stamping process depends on the stress-strain state and the material deformation history. According to the material forming analysis accounting for the springback, its description of tool loading changeability has a distinct effect on the final analysis. The definition of tool and blank sheet interaction being treated as a contact friction of the constitutive model is also used to model the refinement level of the tool elements.

Development of compensation methods based on successive approximation methods with the possibility of defining the measure direction and quality of compensated elements permits an accurate derivation of the unstressed surface. Algorithm of the die correction, with the use of dislocation in the elastic range, implies the iteration procedure of springback compensation [9]. It often happens that optimization can be completed successfully under 
the condition that the entire forming process linked in the correlation chain (forming, trimming, bending and separating operations) will be analyzed (Figs. 1 and 2). The springback mostly occurs after cutting and separating operations. The natural restrictions for application methods of die shape correction are drawpieces, which have vertical walls, because the correction produces tools with wall angles below $90^{\circ}$. It requires special tools with CAM for shaping. Similar restrictions appear for die stampings, which have a large flat surface area $[10,11]$.
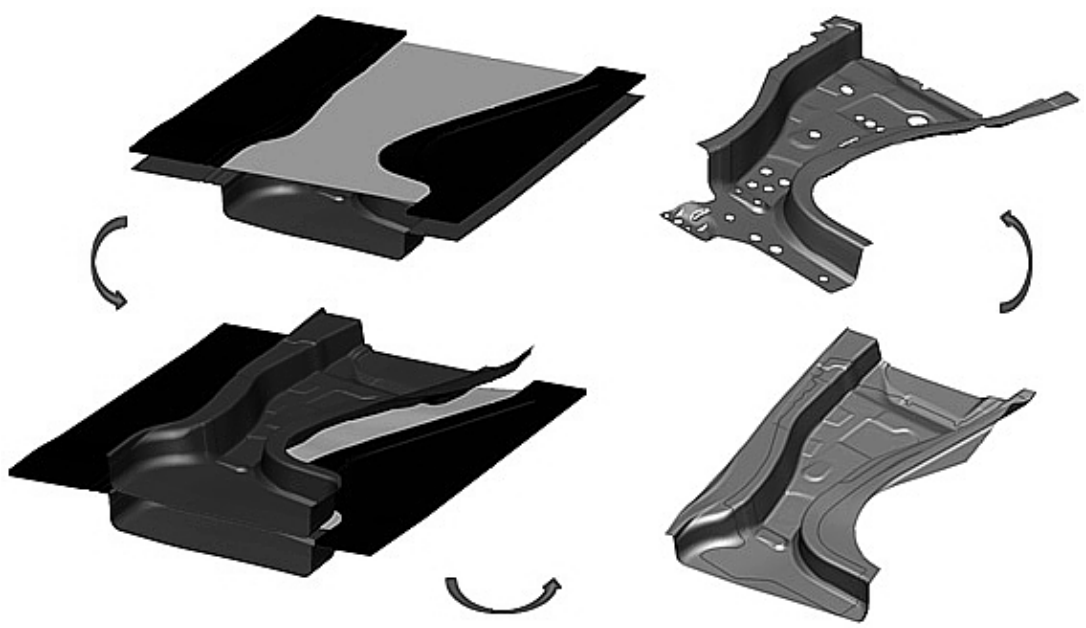

Fig. 1. Visualization of simulation process from forming OP-20 to springback OP-50.

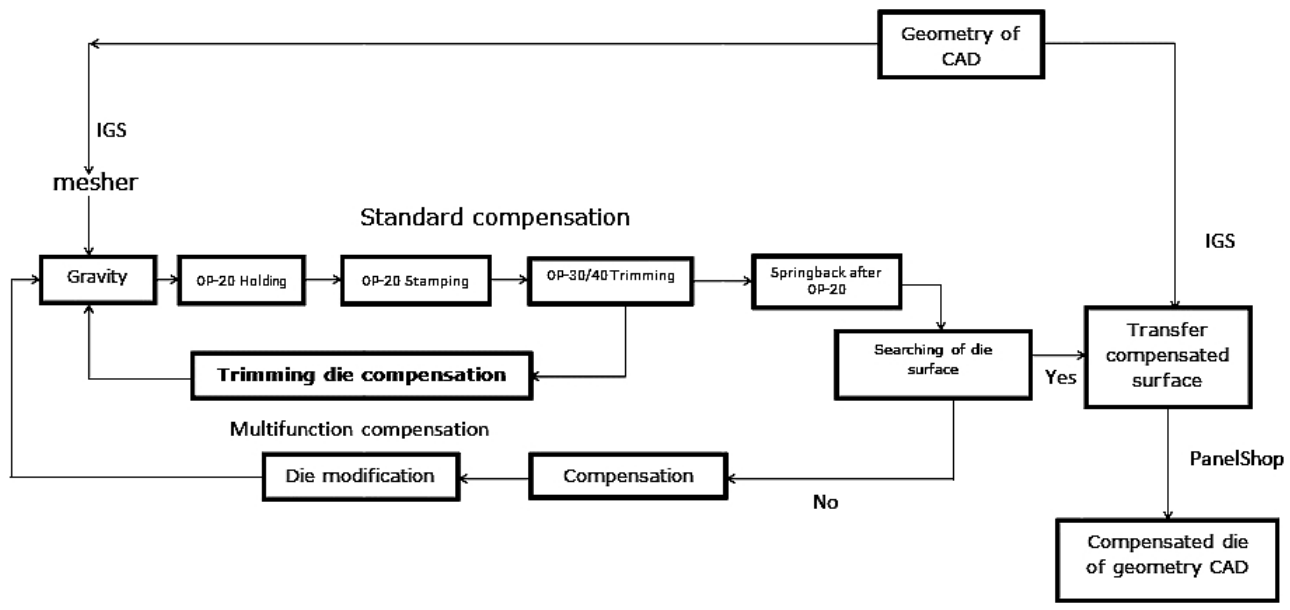

Fig. 2. Compensation process with account of the trimming tool optimization allows one to describe a true forming process.

In multioperation compensation forming, it is necessary to adapt (optimize) the previous tool, in order to compensate the next one. Sometimes, transfer of the forming compensation into the previous operation is used to solve the positioning problems. However, if the dimensional accuracy during the final forming operation is not maintained, the user decides, which operation should be additionally compensated. Usually, two initial forming operations, where most compensation occurs, determine the compensation operation.

It is possible that in OP-20 (forming) and OP30/OP-40 (trimming) there can be an element of compensation, which goes successfully. However, the next forming makes the part different from the reference element. For example, in operation OP-50, there is a 
further springback (Table 1 and Fig. 1). Therefore, influences in the final geometry are not involved in the required tolerance. In order to eliminate the deviation, the compensation is necessary in the subsequent operations. Thus, a good result can be obtained in the particular operations using compensation of subsequent operations.

$\mathrm{T}$ a b 1 e 1

Real Standard Process and Simulated Operations

\begin{tabular}{|c|c|}
\hline True process & Simulated process \\
\hline Gravity & Gravity \\
\hline OP-20 Holding & OP-20 Holding \\
\hline OP-20 Stamping & OP-20 Stamping \\
\hline OP-30/OP-40 Trimming & OP-30/OP-40 Trimming \\
\hline Springback after OP-40 & No springback after OP-40 \\
\hline OP-50 Holding & No OP-50 Holding \\
\hline OP-50 Stamping & OP-50 Stamping \\
\hline OP-60 Trimming & OP-60 Trimming \\
\hline Springback after OP-50 & Springback after OP-50 \\
\hline
\end{tabular}

1.1. Trimming Tool Compensation. Trimming operations usually do not occur individually, but are preceded by a forming operation. This function enables a semiautomatic calculation of stress-relieving in the tool optimization process. On the basis of the calculation results, the surface of the trimming tool gets overbended and the positioning of the drawing parts remains effective. In fact, the surface of the trimming tool has a shape between the calibrated surface and the stress-relieved forming surface, which may lead to some positioning problem with the blank. It is necessary to take into consideration springback transfer from OP-20 (new surface of the forming die) to the tool in operation OP-30/OP-40 (with the overbend for springback in OP-30/OP-40). The account of the trimming tool optimization allows one to simulate a true forming process.

(i) Optimization of the trimming operation improves the efficiency of standard stamping tool compensation and solves the positioning problem of the blank.

(ii) Due to variation in springback of formed parts on the trimming operation, problems with positioning of the blank during transfer from OP-20 (forming operation) to OP-30/OP-40 (trimming operation) may arise.

The innovative compensation method, which additionally includes compensation for trimming stations, is taken into account in the process. A standard compensation method includes only the cutting process during springback calculations, but implies no compensation for the shape of trimming stations (changes in the trimming operation surfaces). The compensated surface of the trimming station should be geometrically embeddedt between the nominal surface of the forming operation and the compensated surface of the same forming operation. In the most cases, for which the springback compensation process is predicted, the surface of the trimming operation is the nominal surface or the compensated one. Using the compensated surface on the trimming and the forming stations increases the accuracy of the whole process. It allows one to get a better compensation result, even more accurate than the standard compensation process. If the compensation is not included at the trimming station, it causes an increase in the reference surface deviation. Furthermore, it also has an influence on the stamped blank positioning errors that appear at trimming station because of the springback difference before and after 
the trimming process. Therefore, it is recommended that this method should be applied to enhance the dimensional tolerance and surface quality. In some particular tools analyzed by the authors, the springback was eliminated by the standard compensation. However, the positioning problem was so difficult that the blank sheet deformation between stations resulted in a distortion, which was visible in the finished element. This led to a search for a compensated surface that was normally separated between operations, which reduced the effect of irregular positioning. Obviously, this situation should be predicted during designing of the tool, in order to avoid extra costs related to reworking of the existing tool and time delays in delivering the prototype elements.

The FEM software that enables optimization of the full process chain is not applicable for generation of the CAD surface due to the fact that such programs generate only a collection of nodes instead of CAD surfaces: this is enough for theoretical purposes but not for industrial conditions. Creation of the stamping die requires milling of each of its parts. Therefore, at the next step it is necessary to convert the mesh in the form of different formats (e.g., Nastran, ANSYS files) into more general CAD formats (e.g., IGS). The process is implemented by PanelShop software [7, 12].

2. Finite Element Model. Taking into consideration the analysis of the part shown in Fig. 3) to find the correct method for trimming tool compensation.

This is a fabricated car element that should ensure safety during side and head-on collisions, especially near a front pillar (Fig. 3). This part is an example of an element, which causes some difficulties due to its geometric specifications and the material used (UHSS).

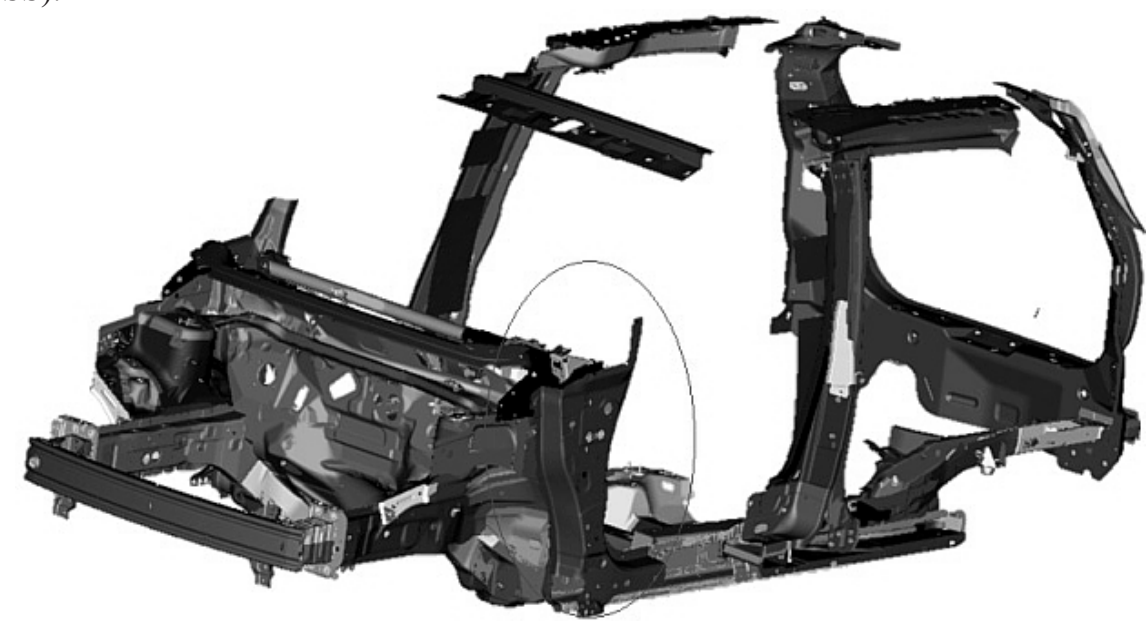

Fig. 3. Body panels with analyzed part A-Pillar.

During design of the forming tool, a lot of problems were encountered, which affected the quality of die stamping, such as excessive local folding, cracking and excessive springback.

The forming process for this part was determined after a few trial simulations and the chosen method (II) was found to be the optimal one.

PAM-STAMP 2G software version 2011.1 [13] was used for computing. It enabled the numerical analysis of the forming process, and complex, full-chain optimization of forming die OP-20 (see Fig. 2), as well as die operation OP-30/OP-40. In OP-20, there are two blank holders (Fig. 4), one for standard holding and a second pad to keep the blank in place during stamping. Operation OP-30/OP-40 is a standard trimming but, actually, this process is subdivided into two operations - OP-30 and OP-40, even though one trimming is accurate enough to achieve a correct simulation. The next process is the second forming 


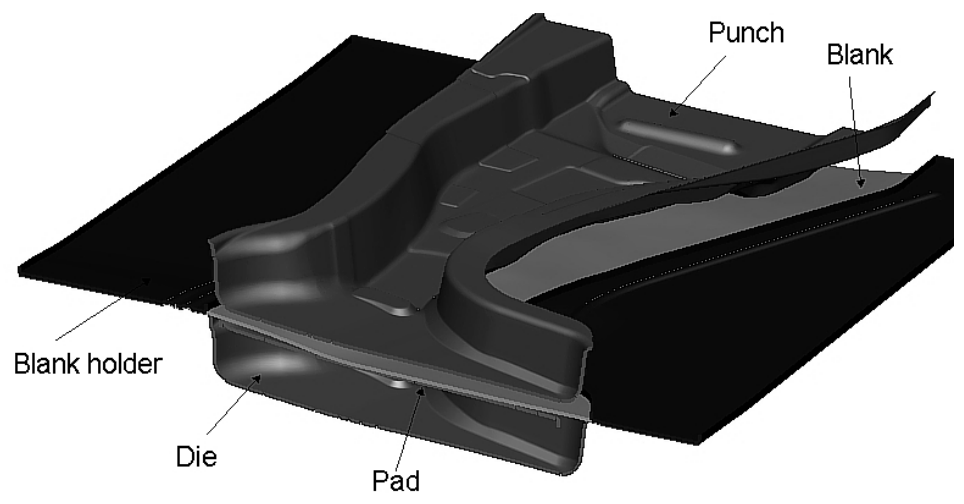

Fig. 4. FEM model with visible areas: die, punch, blank holder, pad and blank.

operation OP-50, which is not included in the FEM analysis due to its small impact on springback.

The finite element numerical analysis made for material model - Hill 48 - explicitly and implicitly uses the computing methods according to the guidelines given by the software suppliers with the aim to minimize computing time and simultaneously maintain a satisfactory accuracy of the server's calculations. There is used Hill 48's law for orthotropic materials, which is applicable to standard HSS steel. The Hill 48 criterion uses the Krupkowsky isotropic hardening law.

As a result, a complex stress state is observed in the blank, which is not always easy for analytical representation via the reduced strength hypothesis. The material used in the forming process has a nominal thickness of $1.5 \mathrm{~mm}$ and the dimensions of the blank are $539 \times 470 \mathrm{~mm}$. For the sake of simulation producing the left and right side parts, a single blank sheet symmetry is assumed, which reduces the computing time. Springback can be also computed taking into consideration the plane symmetry resulting from the cutting operation before the tool calibrationl. The assumption of symmetry makes it necessary to include the boundary conditions, which means that six translations have to be fixed in a statically determined way.

The elements of triangle and quadrangle surface types of nonlinear stress distribution along thickness were used. This is based on the Belytschenko-Tsay model, which implies a uniform, coherent and reduced integration [8]. The thickness integration rule was set to the Gauss rule by default number integration points. Five integration points proved to be sufficient for good accuracy results. The number of elements was optimized relative to the computing time, so that the accuracy reflected the actual conditions. Forming die operation OP-20 contained 53,125 elements and 49,842 nodes, whereas the blank sheet initially contained 5766 elements and 5922 nodes, so the final stage of the process involved about 20,000 elements and 215,255 nodes. Noteworthy is that the number of triangles for the blank sheet equalled to 186 at the end of the process. However, the number of quadrangles is variable: at the beginning the amount was 5766, whereas at the end it was 205,815 .

3. Material Model. Applied DP-600 material is a double-phase cold-rolled.steel that belongs to the HSS group. The steel structure is formed by the compound in die form of martensite precipitates on fine-grained ferrite warp. There also may appear small amounts of retained austenite. These steels are produced by suitably provided cooling after annealing from the temperature range permitting co-existence of $\alpha$ and $\gamma$ phases. The properties of this steel (which is a kind of specific composite) are the result of combining portions of hard and resistant martensite and ductile ferrite. It provides a high strength and extension of several percents (Table 2). There is also a high initial yield point and ultimate strength that quickly declines during the plastic working. Hill 48 material using isotropic 
$\mathrm{T}$ a b 1 e 2

Mechanical Properties DP-600 for Various Rolling Directions

\begin{tabular}{|c|c|c|c|c|c|c|c||}
\hline $\begin{array}{c}\text { Test } \\
\text { direction } \\
(\mathrm{deg})\end{array}$ & $\begin{array}{c}h, \\
\mathrm{~mm}\end{array}$ & $\begin{array}{c}R_{p 0.2}, \\
\mathrm{MPa}\end{array}$ & $\begin{array}{c}R_{m}, \\
\mathrm{MPa}\end{array}$ & $\begin{array}{c}A_{50 \mathrm{~mm}}, \\
\%\end{array}$ & $\begin{array}{c}A_{80 \mathrm{~mm}}, \\
\%\end{array}$ & $\begin{array}{c}r, \\
10 \%\end{array}$ & $\begin{array}{c}n, \\
5-10 \%\end{array}$ \\
\hline 0 & 1.22 & 433 & 634 & 29 & 24 & 0.73 & 0.16 \\
\hline 45 & 1.24 & 446 & 626 & 31 & 25 & 1.09 & 0.16 \\
\hline 90 & 1.23 & 454 & 636 & 29 & 24 & 0.83 & 0.16 \\
\hline
\end{tabular}

hardening curve was applied despite the fact that the software uses the criterion of the isotropic-kinematic consolidation hypothesis.

The chosen material has the following basic material parameters: the Young modulus $E=210 \mathrm{GPa}$, Poisson's ratio $v=0.3$, density $\rho=7800 \mathrm{~kg} / \mathrm{m}^{3}$, rolling direction along the shorter edge of a blank sheet (local $x$ axis), and blank thickness $g_{0}=1.2 \mathrm{~mm}$. Normal anisotropy can be expressed by $r_{\alpha}$ in direction:

$$
r_{\alpha}=\frac{\varepsilon_{2}}{\varepsilon_{3}}=\frac{\ln \left(b / b_{0}\right)}{\ln \left(g / g_{0}\right)},
$$

where $\alpha$ is direction, $b$ is width, and $g$ is thickness of the specimen, while $b_{0}$ and $g_{0}$ are the respective initial values $[14,15]$. The reference parameter functions $(G, H, F, N)$ are expressed by anisotropy coefficients for characteristic rolling directions 0,45 , and $90^{\circ}$ (also referred to as the Lankford coefficients),

$$
F=0.41, \quad G=0.56, \quad H=0.44, \quad N=1.59 .
$$

The coefficient describing normal anisotropy $r$ is regarded as the average value that can be determined from the following formula:

$$
r=0.25\left(r_{0}+2 r_{45}+r_{90}\right)=0.25(0.77+2 \cdot 1.14+1.07)=1.03 .
$$

Hardening curve determined by static tensile test for $0^{\circ}$ direction after calculating stress value and actual strain. Next, the curve is extrapolated, in order to increase the range of actual strain [16]. To achieve this, the Krupowsky formula was used:

$$
\sigma_{w}=K\left(\varphi+\varphi_{0}\right)^{n}=0.6908(\varphi+0.008798)^{0.1494} .
$$

The hardening curve is not sufficient for checking puposes during forming analysis: the forming limit diagram (FLD) must be used additionally for accurate verification of the nodes, which are critical in terms of the maximal stress.

This diagram of steel drawability is constructed with the use of main straining. The curve can be determined by the Nakajima test or by other experimental methods $[14,17]$.

4. Numerical Parameters for Springback Compensation. The blank sheet is treated by default as the middle layer without thickness. Contact between the die and the blank was adopted as automatic. In OP-20, the blank holder was used, in order to reduce wrinkling.

The defined forces equal relatively $400 \mathrm{kN}$. The speed of the blank holder has been defined as $2 \mathrm{~m} / \mathrm{s}$, while the speed of forming as velocity balanced curve (VBC) with the maximal value of $5 \mathrm{~m} / \mathrm{s}$. It is necessary to define the velocity scale factor for variable rate 
that is set as $v c c=0.1$. If the punch and blank-holder velocity is multiplied by scale factor, the simulation model satisfies that condition. It is required to provide the velocity correction in advance parameter attributes of the global object, which accounts for the relationship between the simulation time $t$ and physical time $T$.

Modeling of contact that is based on the method of penalty friction coefficient between complementary surfaces is described as Coulomb and dynamic coefficient friction or the function depending on the variables of the other parameters, e.g., stamping velocity, pressure force, etc. The friction model as the Coulomb coefficient is a bilinear model, which uses static (i.e., defined as constant value) $\mu=0.11$ [8]. The level of refinement of the mesh is based on the criterion of the minimal radius value $r=3$, which enforced refinement degree at level 5, while the minimum element value is $1.21 \mathrm{~mm}$.

5. Springback Prediction and Compensation. In design of cold forming tools, the springback effect should be definitely taken into account, in view of the material used and the complicated geometry. In addition to the internal stresses that arise as a result of bending, there is a residual stress distribution in the cross section of bent material. This residual stress distribution depends on the extent to which the material underwent plastic deformation. Even though the springback is occasionally more or less visible depending on the type of material, it is a result of bending of the material. For parts with no complex geometry, the analytic methods for solution are advisable for rapid size estimation, whereas complex structures of complicated shapes require numerical solution of the problem.

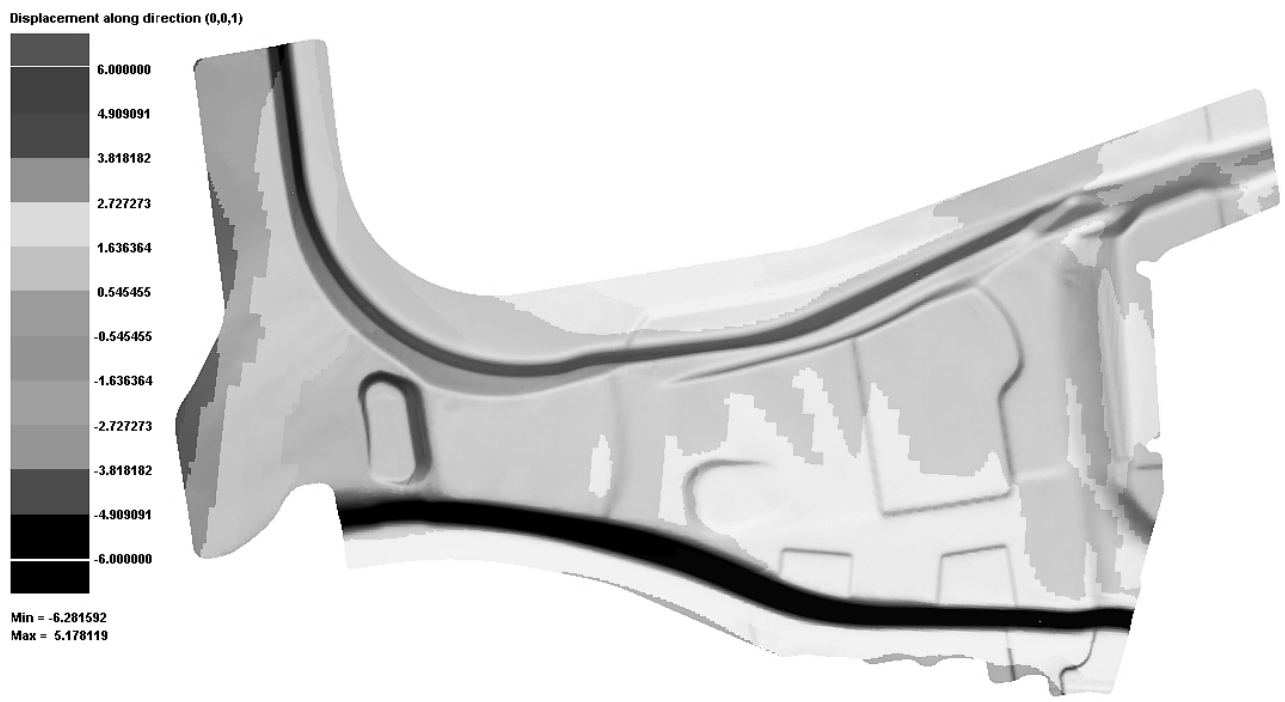

Fig. 5. Springback deviation computed by FEM.

The accuracy of springback prediction also depends on the influence of coefficients, which are responsible for forming analysis quality. Initially, the maximum springback value was assumed as about $6 \mathrm{~mm}$ (Fig. 5) in the direction of the local $z$ axis. It is possible to estimate the scale of the springback by calculating the value in an analytic way $[14,18,19]$ :

$$
\Delta \alpha=0.0143 R_{m}^{0.94}\left(R_{i} / t\right)^{0.1}=0.0143 \cdot 600^{0.94}(6 / 1.2)^{0.1}=6.86(\mathrm{deg}) .
$$

6. Finite Element Analysis. The conducted analysis of stress state accepts the level below $20 \%$ of thinning according to customer requirements, although initially there was found to be a major tendency for the material superposition and availability of an area of crack propagation. The change of geometry of OP-20 clamp surface and optimization of 
blank-holder force enabled adjustment for the most proper process parameters. After optimization the forming process is stable without any cracks or strong wrinkling tendency.

7. Experimental Verification. As it was numerically and experimentally proved, springback values reached the assumed level of about $6 \mathrm{~mm}$ for OP-20 (Fig. 5). The numerical analysis showed that the initial tool compensation without full chain compensation brought an unsatisfactory result. The deviation value after compensation is above $1.5 \mathrm{~mm}$, which vastly exceeded the complex tolerance range (Fig. 6). It is caused by optimizing only OP-20 (forming) without taking into account OP-30 (cutting) (Fig. 1). During the trimming operation a small-scale deformation of unstressed parts occurred, which produced parts that failed to meet the requirements of imposed tolerance $\pm 0.8 \mathrm{~mm}$. However, the application of

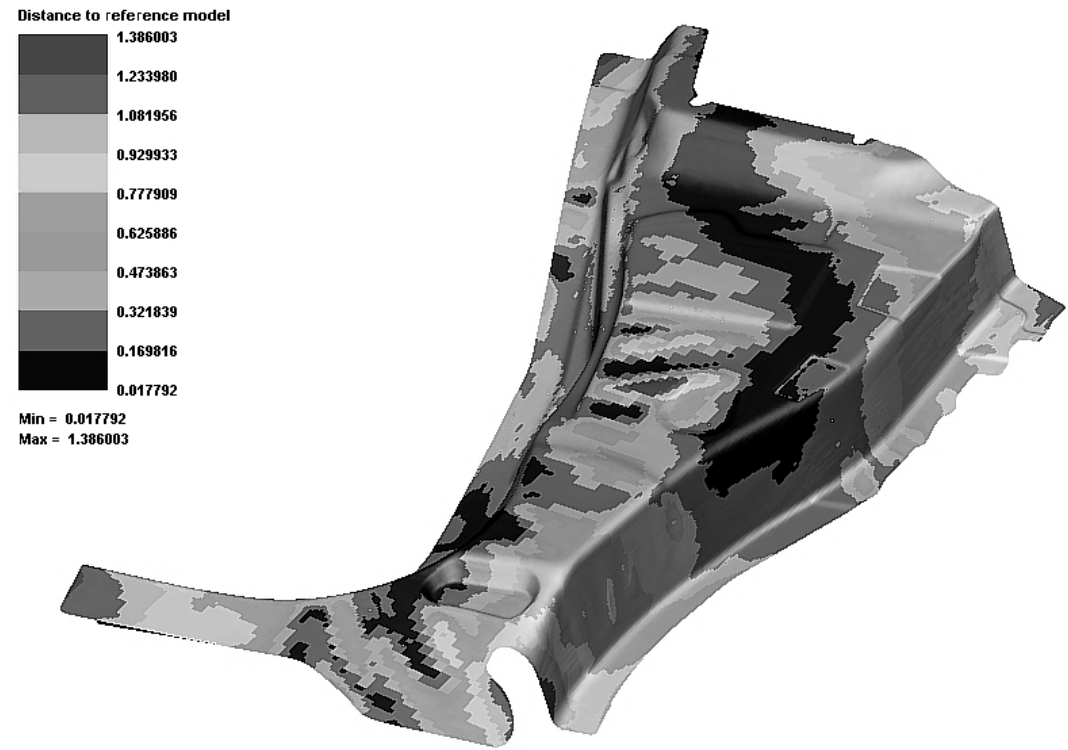

Fig. 6. Distance to the reference part after compensation without full chain.

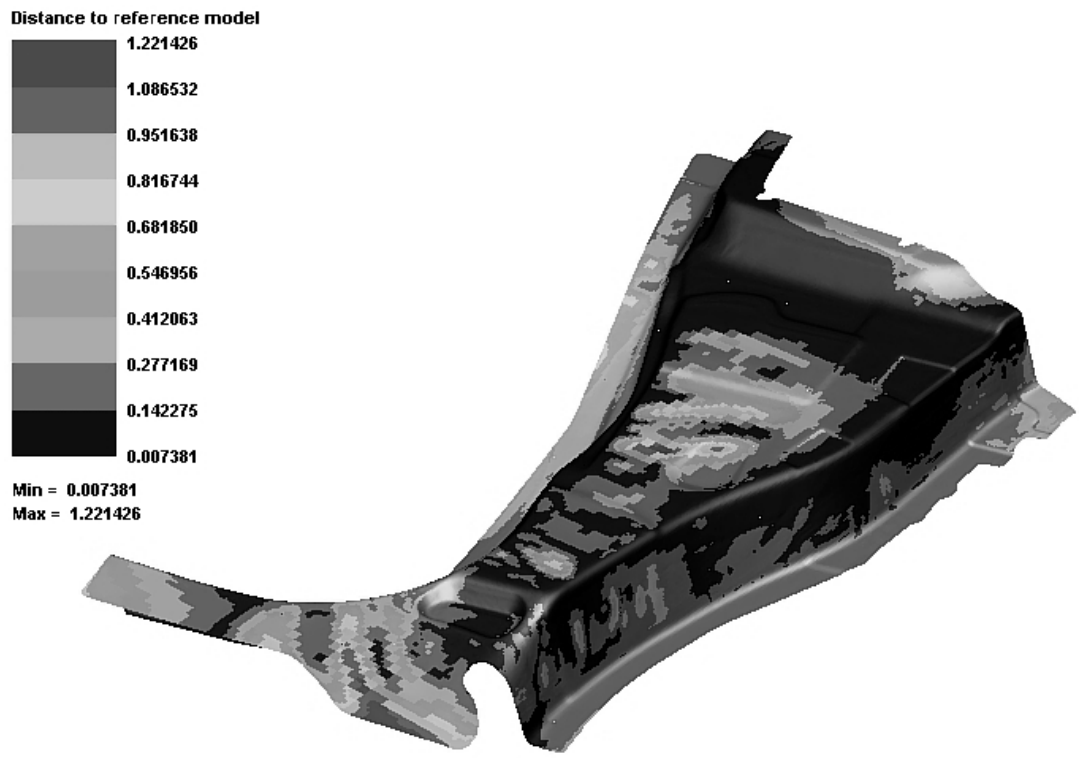

Fig. 7. Distance to the reference part after compensation with full chain. 


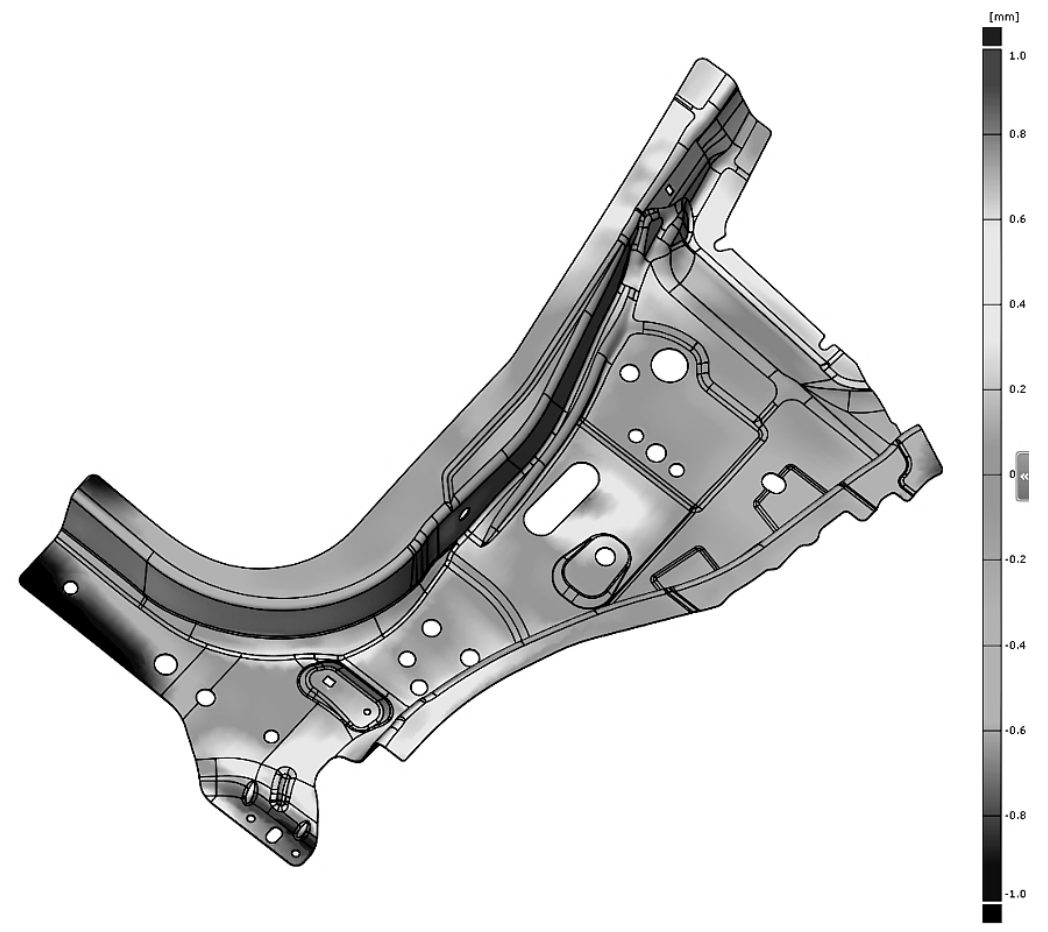

Fig. 8. Measured part formed with multicompensation by optical measuring techniques. (Maximum deviation is $\pm 1.2 \mathrm{~mm}$ on surface.)

the complex springback compensation enabled production of satisfactory parts, which were within the tolerances required by the customer (Fig. 7). It must be emphasized that some parts' zones are less important and may only fit into general tolerances, which are usually wider than those for mating zones and are equal to $\pm 1 \mathrm{~mm}$ (Fig. 8). Sometimes, a larger springback tolerance is also allowed in the spot of the next forming and in that way it is used in the presented part. The zone with the deepest overpress is formed during another OP-50 operation and therefore, compensation with lower tolerance is acceptable.

Conclusions. New materials, such as UHSS, have a high structural potential, but their application requires implementation of novel technologies in the rework process of these materials. More and more intensive economic competition and an increase in expectations for the final product demand increased efficiency in a stamping department. Customer quality expectations generally revolve around appearance, security, comfort and low fuel consumption. This is also related to visible elements - for example, outer element coverage and pillars, which also have esthetic functions that cannot accept any geometric alterations.

Using curvilinear surfaces in components is meant to get smooth reflections so the formed surface contains no surface defects. The forming process of materials with high mechanical parameters requires the complex compensation because such materials are highly prone to springback effect, which is not always easy to eliminate. In regard to tightening geometric tolerance requirements, compensation of springback effect should meet the increasingly high customer expectations. Therefore, improved methods for reducing springback are being sought.

As the numerical analysis and physical experiment confirmed, a failure to account for the springback during forming process yields defected parts that do not comply with the requirements.

The following factors need to be taken into consideration to get a compensated surface that will meet the assumed tolerance: 
1. Accurate material data taking into account the hardening curve and FLD chart.

2. Correct forming process with no cracks or strong wrinkling.

3. Meshing strategy as compensation (including the criterion of the maximal angle, which must be set as $7.5^{\circ}$ ). scale).

4. Defining of punch velocity as a ramp with a maximum speed of $5 \mathrm{~m} / \mathrm{s}$ (including

5. Use of correct boundary condition strategy (set statically or manually determined by RPS points).

6. Correct computing solver type (implicit, explicit, advance implicit).

Firstly, the most important aspect is accuracy of simulation that allows for exact data concerning material, boundary conditions and process parameters. The material model is so important that it should take into account material data of the specific supplier even during springback calculation. Compensation is determined so much by springback calculation accuracy that if it is not ehigh enough, achieving the required result will be, in practice, impossible.

The breakthrough in compensation quality has occurred with introduction of the multioperation compensation. It has enabled a better simulation of true processes by correction/adjustment of individual tooling stations, which has increased the precision of computation.

The reached compensated surfaces of forming and cutting operations at the design stage imply considerable time saving and, especially, cost reduction, as compared to the trial-and-error method. These kinds of parts with such complex geometry and material specification require approximately a few months of hard work, in order to reach the assumed geometry that is contained in the tolerance range. Drawpieces' defects such as cracks and excessive folding are predicted and avoided by using well-known procedures, but application of the latter for the springback effect account often remains problematic without reliable data on the true operation environment.

1. H. P. Vogt, Modern Lightweight Design with Magnesium Flat Products, Magnesium Flachprodukte GmbH, Freiberg (2012).

2. Ultra-High-Strength Steels, Enhancing Vehicle Safety and Improving Fuel Efficiency: media.ford.com, 07/2011.

3. N. Seki, Successfully Controls Springback in Thin Ultra-High Strength Steel Stamping Parts Using PAM-STAMP 2G, TOA Industries (2011).

4. High-Strength Steels for Shallow Formed Part, ThyssenKrupp Steel, Duisburg, (2009).

5. S. S. Kang and D. H. Park, "Application of computer-aided process planning system for non-axisymmetric deep drawing products," J. Mater. Process. Technol., 124, 36-48 (2002).

6. J. C. Choi, C. Kim C., Y. Cho, et al., "An integrated design and CAPP system for deep drawing or blanking products," Int. J. Adv. Manuf. Technol., 16, 803-813 (2000).

7. J. Danzberg and S. Schmaalze, Parameterization of the Tool Geometry as a Prerequisite for the Shape Optimization of Tool Surface, Zurich (2007).

8. Atlas Tool Inc., Develops a Sounds Springback Compensation Process with the Help of Pam-Stamp $2 G$ Software, ESI Group (2011).

9. H. Wei, W. Chen, and L. Gao, "Springback Investigation on sheet metal incremental formed parts," Int. J. Mech. Aerosp. Industr. Mechatr. Manuf. Eng., 5, No. 7, 12731277 (2011). 
10. D. Bekar, E. Acar, F. Ozer, and M. A. Guler, "Robust springback optimization of DP600 steels for U-chanel forming," in: Proc. of the World Congress on Engineering (July 6-8, 2011, London), Vol. III, Newswood Limited (2011), pp. 2399-2404.

11. J. Gronostajski, A. Niechajowicz, and S. Polak, "Springback deformation of drawpieces after trimming and parting," in: Umformtechnik Tagungsband "Werkstoffe und Komponenten für den Fahrzeugbau" (06-08.10.2004, Freiberg), Institut für Metallformung TU Bergakademie (2004), pp. 457-476.

12. PanelShop iCapp Automated Springback Compensation, User's Guide, version 11.0, Zurich (2011).

13. ESI Group PAM-STAMP 2 G 2011, User's Guide, Paris (2011).

14. H. Lundh, P. A. Bustad, B. Carlsson, et al., Sheet Metal Forming, SSAB Tunnplant, Goteborg (1998), pp. 2:1, 2:2, 4:3, 4:4, 4:10, 4:17, 5:14.

15. U. Bergstrom, M. Bronnestam, L. Gustafsson, et al., "Design and fabrication in high strength steel," in: Sheet Steel Handbook, SSAB Tunnplant AB, Borlange (1996), pp. 5:12-5:27.

16. M. Firat M., B. Kaftanoglu, and O. Eser, "Sheet metal forming analysis with an emphasis on springback deformation," J. Mater. Process. Technol., 196, 135-148 (2008).

17. C. Leppin, J. Li, D. Daniel, "Application of a method to correct the effect of nonproportional strain paths on Nakajima test based forming limit curves," in: Proc. of the 7th Int. Conf. and Workshop on Numerical Simulation of 3D Sheet Metal Forming Processes (NUMISHEET, Interlaken, Switzerland), ETH Zurich (2008), pp. 217-221.

18. D. A. Smith, Die Design Handbook, Society of Manufacturing Engineers, Princeton, NJ (1990), pp. 6-15-6-16.

19. I. Suchy, Handbook of Die Design, McGraw-Hill, New York (1997), pp. 8-28-8-33.

Received 10. 08. 2016 